\title{
Radial velocities of Hipparcos southern B8-F2 type stars $^{\star, \star \star}$
}

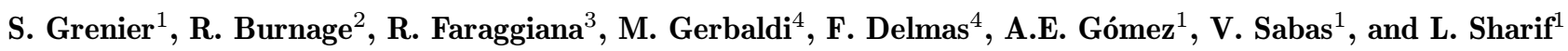 \\ 1 Observatoire de Paris-Meudon, DASGAL, URA 335 du CNRS, F-92195 Meudon Cedex, France \\ 2 Observatoire de Haute Provence, UPR 9049 du CNRS, F-04870 Saint Michel l'Observatoire, France \\ 3 Dipartimento di Astronomia, v. Tiepolo 11, 34131 Trieste, Italy \\ ${ }^{4}$ Institut d'Astrophysique de Paris, CNRS, 98bis Bd. Arago, F-75014 Paris, France
}

Received October 21; accepted November 19, 1998

\begin{abstract}
Radial velocities have been determined for a sample of B8-F2 type stars observed by the Hipparcos satellite. Observations were obtained within the framework of an ESO key-program. Radial velocities have been measured using a cross-correlation method, the templates being a grid of synthetic spectra. The obtained precision depends on effective temperature and projected rotational velocity of the star as well as on a possible asymmetry of the correlation peak generally due to secondary components. New spectroscopic binaries have been detected from these asymmetries and the variability of the measured radial velocity. Simulations of binary and triple systems have been performed. For binaries our results have been compared with Hipparcos binary data. Adding the variable radial velocities, the minimum binary fraction has been found $60 \%$ for physical systems. Radial velocities have been determined for 581 B8-F2 stars, 159 being new. Taking into account published radial velocities, $39 \%$ south A-type stars with $V$ magnitude lower than 7.5 have a radial velocity.
\end{abstract}

Key words: techniques: spectroscopic — stars: earlytype — stars: fundamental parameters — binaries: spectroscopic

\section{Introduction}

The Hipparcos mission, active from November 1989 to March 1993, measured very precise astrometrical data

Send offprint requests to: R. Burnage

* Based on observations obtained at the European Southern Observatory (ESO, La Silla, Chile) and on data from the ESA Hipparcos astrometry satellite.

** Tables 7, 8 and 9 are only available in electronic form at the CDS via anonymous ftp to cdsarc.u-strasbg.fr (130.79.128.5) or via http://cdsweb.u-strasbg.fr/Abstract.html for 118000 stars. These results are published in The Hipparcos Catalogue (ESA 1997). Up to the Hipparcos magnitude $H_{\mathrm{p}}$, the median precision of positions, parallaxes and proper motions are better than 1 mas and 1 mas $\mathrm{yr}^{-1}$ respectively.

An ESO key-program (Gerbaldi et al. 1989) was allocated to obtain complementary radial velocity data as well as fundamental parameters for early-type stars nearer than 100 pc observed by the Hipparcos satellite. The major aims were the detailed study of the HR-diagram, in particular the calibration of the absolute magnitude $M_{v}$ and the determination of age and mass in order to study the kinematics and evolution of these stars. A first determination of $M_{v}$, age and mass of these stars has been made by Sabas (1997) and applied in Sabas et al. (1998). The same spectra have been used for a detailed independent analysis of the A0-type stars performed by Gerbaldi et al. (1998), giving the determination and the discussion about the effective temperature $\left(T_{\text {eff }}\right)$ and the gravity $(g)$. The projected rotational velocity $(v \sin i)$ has been determined by Royer et al. (1998). We limited the sample to normal stars between B8 and F2 and luminosity classes V to III, excluding known chemically peculiar stars.

Radial velocities have been obtained using a crosscorrelation method with synthetic spectra. Their precision strongly depends on $v \sin i$ and $T_{\text {eff }}$ of the star. New binaries have been detected from the analysis of the shape of correlation peaks and from the variable radial velocities. These results, which have been compared with Hipparcos data about double and multiple systems, are often complementary in terms of separation, magnitude difference and orbital period.

\section{Observations and data reduction}

\subsection{Observational program}

The aim of the ESO key-program required large wavelength range to allow to detect possible peculiarities, 
a 20000 minimum resolution for $v \sin i, T_{\text {eff }}, \log g$ and metallicity determinations and an accessible instrumentation several times a year. These conditions were met by the Echelec Spectrograph equipped with a CCD camera at the $1.52 \mathrm{~m}$ ESO telescope at La Silla. Priority was given to radial velocities determinations. The initial program of B8-F2 stars nearer than $100 \mathrm{pc}$ changed with time, the lack of transparency of the instrumentation not allowing the observation of stars fainter than $V$ magnitude 7.5. So the number of F-type stars was reduced and priority was given to early A-type stars. Reference stars were observed for the different researched parameters. Some supergiants were added to obtain their $v \sin i$. We observed, only once, stars for the obtention of $v \sin i$ and twice for radial velocity. Meanwhile it appeared interesting to determine radial velocity for the whole sample, even when there was one observation only. Keeping in mind that Hipparcos Catalogue is complete until $V$ magnitude 7.5, it contains 3545 south normal stars from B8 to F2 and $V<7.5,58 \%$ of which being A-type, and $39 \%$ having a radial velocity now. As a result, 610 stars were observed during 21 observation runs, from June 1989 to January 1995, 581 being in the range $\mathrm{B} 8$ to $\mathrm{F} 2$ type, $41 \%$ of which having two or more measurements.

\subsection{Instrumentation}

The ESO Echelec spectrograph was used at the coudé focus of the $1.52 \mathrm{~m}$ telescope at La Silla (Chile). The 9 central orders around $\lambda_{\mathrm{c}}=4350 \AA$ were used. They covered the spectral range 4210 to $4500 \AA$. The dispersion was $3.1 \AA \mathrm{mm}^{-1}$. The detector was a RCA CCD with $640 \times 1024$ pixels of $15 \times 15 \mu \mathrm{m}^{2}$, the pixel size corresponding to $0^{\prime \prime} 65$ on the sky. The nominal spectrograph resolution was about 70000 . The characteristics are described in detail in Gilliotte \& Lindgren (1989). Using a slit width of $320 \mu \mathrm{m}$ (1'.5 on the sky), the instrumental resolving power was degraded to 28000 and the ratio $\mathrm{S} / \mathrm{N}$, highly variable from the center to the edges of each order covered the range 50 to 200 . The reduction from a CCD image to a complete linear spectrum (calibration frames, orders extraction, wavelength calibration, connection of the orders) is described with details by Burnage \& Gerbaldi (1990, 1992).

\subsection{Correlation method}

A cross-correlation method with synthetic spectra was chosen to determine radial velocities. This method, described by Tonry \& Davis (1979), to determine galaxy redshifts was since used by several authors for late-type stars, but not often for early-type stars. Main difficulties in A-type stars come from the small number of lines and the fact that the $\mathrm{H} \gamma$ line covers most of the spectral range of the spectra at our disposal. It would contribute far too much in the computation of a correlation index with respect to the faint metallic lines more or less washed out by the high projected rotational velocity of most stars. In order to use metallic lines only, a pseudonormalised spectrum has been computed whose continuum follows the profile of $\mathrm{H} \gamma$. So the correlation was independent of this line. A programme of automatic normalisation has been elaborated and applied.

Synthetic spectra have several advantages compared to actual stellar spectra for the use as templates: they are perfectly adapted to the linear response of CCD detector; they are noise-free; their radial velocity is zero and they allow to compute an homogeneous and regular grid of reference spectra. They do not incorporate the instrumental profile, whose influence is negligible on the spectra of these mean-high rotating stars. They were computed with Kurucz (1993) models. The range of $T_{\text {eff }}$ was 6000 to $15000 \mathrm{~K}, \log g=4.0$ and metallicity solar (justified by the sample of normal stars) and lines were widened by rotation with a path of $25 \mathrm{~km} \mathrm{~s}^{-1}$. The resolution of synthetic spectra was adapted to the observed spectra (same step and start). As to avoid the differential dispersion due to the radial velocity, all spectra were rebinned in Naperian logarithms.

All reduction programs were built into the MIDAS environment. Systematically the correlation program identified the synthetic spectrum giving the best correlation index (see the description of the grid of the synthetic spectrum in the Sect. 3.1). This method allowed to test the spectral types and rotation velocities found in the literature. Discrepancies came from binaries generally. The range of correlation index varied from 0.5 to 0.95 in most cases. The maximum of the cross-correlation function which was usually quasi Gaussian was estimated by fitting its peak with a parabola.

\subsection{Zero point of radial velocities}

The zero-point was determined from IAU standard which were observed every night, when it was possible. To have an homogeneous determination, we adopted the radial velocities obtained with Coravel and given by Latham \& Stefanik (1991). The zero-point was determined for each run. The result on IAU standards is summarized in Table 1, where $V r_{\text {cor }}$ is the Coravel value, $V r_{\text {ech }}$ the mean observed value obtained with spectra with its rms (root mean square) and $n$ the number of spectra.

The independence of obtained radial velocities with the spectral type was tested using the homogeneous radial velocities of B to F type stars from Andersen \& Nordström (1983) and Nordström \& Andersen (1985) (see the comparison in the Sect. 3.3). 
Table 1. IAU standards used for zero-point

\begin{tabular}{rrrrr}
\hline HD & $V r_{\text {cor }}$ & $V r_{\text {ech }}$ & rms & $n$ \\
\hline 693 & 14.92 & 13.65 & & 1 \\
4128 & 12.71 & 12.77 & 0.44 & 11 \\
22484 & 27.85 & 27.56 & 0.42 & 19 \\
36079 & -14.37 & -14.35 & 0.49 & 26 \\
66141 & 71.14 & 71.36 & 0.53 & 3 \\
81797 & -5.31 & -5.07 & 0.36 & 19 \\
109379 & -7.86 & -7.99 & 0.54 & 33 \\
136202 & 54.30 & 54.46 & 0.38 & 5 \\
157457 & 17.22 & 17.04 & 0.04 & 2 \\
161096 & -12.86 & -12.23 & 0.26 & 6 \\
168454 & -20.84 & -20.55 & 0.60 & 10 \\
171391 & 7.10 & 7.31 & 0.38 & 4 \\
182572 & -100.33 & -100.45 & 0.48 & 2 \\
203638 & 21.65 & 21.48 & 0.59 & 3 \\
204867 & 6.02 & 5.90 & 0.44 & 12 \\
212943 & 53.85 & 53.99 & 0.39 & 5 \\
222368 & 5.58 & 5.21 & 0.16 & 2 \\
\hline
\end{tabular}

\subsection{Correlation peaks analysis}

Generally the correlation peak is symmetric and roughly Gaussian. Nevertheless some of them were double, asymmetric or not Gaussian and their interpretation was not obvious. To detect an eventual asymmetry, each peak was fitted with a Gaussian profile and a new radial velocity was calculated (the parabola only fits the top of the peak whereas the Gaussian fits the whole profile). The difference $d v_{\text {gp }}$ of this new radial velocity with the value obtained with a parabola was computed. Several cases, function of the shape of peaks, are considered. Separated peaks of binaries are listed in Table 7 with a and b components. Some radial velocities were considered as not significant: when the correlation coefficient was lower than 0.3 or when the peak was too asymmetric (detected by a Chi-Square test upon $d v_{\mathrm{gp}}$ ). The Hipparcos (HIP) and HD numbers of these stars are listed in Table 5 . Symmetric but wide peaks could mask a binary system. To analyse the shape of some peaks, simulations of binary and triple systems were made with synthetic spectra.

In the simulations, the input parameters were $T_{\text {eff }}$ and rotation broadening of components, their radial velocities difference and the ratio of their intrinsic luminosities. It was not possible to do this simulation for each peak but this has allowed to recognize characterized shapes of binary peaks. With the aim of detecting a possible double or multiple system, hidden by rotation, all spectra were correlated with the minimum rotation available in our grid of synthetic spectra. A number ranging from 0 to 10 refers to the shape of the corresponding correlation curve in Tables 8 and 9. The meaning of this flag is summarized in Table 4 . The agreement between $T_{\text {eff }}$ and rotation

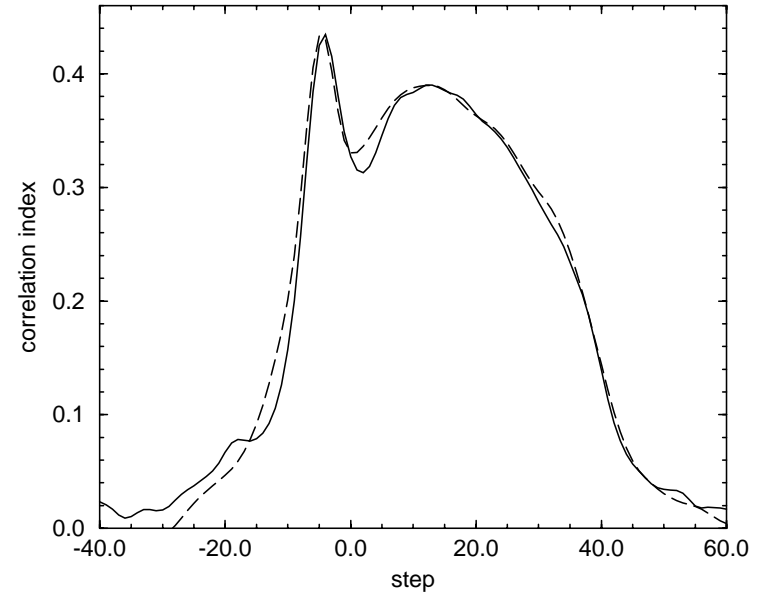

Fig. 1. Simulation (in dashed line) of the double correlation peak of HD 6668 (in full line)

broadening of synthetic spectra found for the different observed spectra of a same star is considered: in $79 \%$ stars these parameters are the same. For the others, $13 \%$ show a duplicity criterion and in the remaining $8 \%$ the real values are between the two found values, probably.

\section{Data analysis}

\subsection{The internal precision}

The different error origins are the zero-point for each observing run, the choice of synthetic spectra and a possible peculiarity of the star (shell, binary or multiple), the noise of CCD being negligible. The zero-point error was determined as described in Sect. 2.4. The error on the radial velocity arising from the choice of the synthetic spectrum depends on $v \sin i$ and $T_{\text {eff }}$ : it is lower than $0.1 \mathrm{~km} \mathrm{~s}^{-1}$ for $v \sin i \leq 25 \mathrm{~km} \mathrm{~s}^{-1}$, about $1.0 \mathrm{~km} \mathrm{~s}^{-1}$ for $v \sin i=125 \mathrm{~km} \mathrm{~s}^{-1}$, and gets $9 \mathrm{~km} \mathrm{~s}^{-1}$ for $v \sin i=250 \mathrm{~km} \mathrm{~s}^{-1}$ and $T_{\text {eff }}=12500 \mathrm{~K}$. This estimation is made by correlation between two adjacent synthetic spectra for every possible combinations and is given in Table 2. Another error depends on the shape and symmetry of the peak. Its estimation is computed by $d v_{\text {gp }}$ as described in Sect. 2.5 and given in Table 7. When the shape of the peak is clearly too asymmetric and irregular, the radial velocity is not given. HIP and HD numbers of these stars are given in Table 5. Finally we adopt as estimation of internal error $\sigma_{i 0}$ :

$\sigma_{i 0}^{2}={\sigma_{\text {run }}}^{2}+\sigma_{\text {mask }}^{2}+d v_{\text {gp }}^{2}$

$\sigma_{\text {run }}$ is the rms of the zero-point of each run, $\sigma_{\text {mask }}$, depending on synthetic spectrum, is given in Table 2 and $d v_{\text {gp }}$ gives an estimation of the asymmetry of the peak. To check the reality of this estimation, the unit-weight error was computed: for each star having several measurements $v_{i}$, the mean radial velocity $\bar{v}$ was computed and the distribution of the values $\left(v_{i}-\bar{v}\right) / \sigma_{i 0}$ has shown that the 
Table 2. Error on the radial velocity in $\mathrm{km} \mathrm{s}^{-1}$ arising from the choice of the parameters $T_{\text {eff }}$ in $\mathrm{K}$ and rotation in $\mathrm{km} \mathrm{s}^{-1}$ in synthetic spectra

\begin{tabular}{lllllllll}
\hline $\begin{array}{l}T_{\text {eff }} \\
\text { rot }\end{array}$ & 6000 & 6500 & 7500 & 8500 & 9200 & 10000 & 11000 & 12500 \\
\hline 5 & 0.00 & 0.00 & 0.00 & 0.01 & 0.01 & 0.01 & & \\
10 & 0.00 & 0.00 & 0.01 & 0.02 & 0.02 & 0.02 & 0.02 & 0.02 \\
$25 / 30$ & 0.01 & 0.01 & 0.01 & 0.03 & 0.03 & 0.03 & 0.04 & 0.06 \\
50 & 0.02 & 0.02 & 0.02 & 0.04 & 0.05 & 0.10 & 0.12 & 0.14 \\
75 & & & 0.06 & 0.08 & 0.09 & 0.22 & 0.38 & 0.5 \\
100 & & 0.10 & 0.10 & 0.14 & 0.18 & 0.41 & 0.8 & 0.9 \\
125 & & & & 0.22 & 0.28 & 0.9 & 1.4 & 1.5 \\
150 & & & 0.26 & 0.33 & 0.40 & 1.2 & 2.0 & 2.2 \\
175 & & & & 0.45 & 0.56 & 1.6 & 2.8 & 3.0 \\
200 & & & & 0.6 & 0.8 & 2.3 & 4.0 & 4.2 \\
250 & & & & & & 3.4 & 7.2 & 8.6 \\
\hline
\end{tabular}

estimation of $\sigma_{i 0}$ was slightly pessimistic. Subsequently, the given internal error $\sigma_{i}$ has been adopted as $0.85 \sigma_{i 0}$.

\subsection{Comparison with classical method}

The radial velocities obtained by correlation were compared with those obtained by the classical method of individual measurements of spectral lines upon a part of the same spectrum. In the classical method in which the internal error estimation is strongly function of the $v \sin i$ of stars, the number of measured lines decreases quickly with the projected rotational velocity. Comparable internal rms are found for stars with small $v \sin i$, the correlation method becoming quickly more accurate for $v \sin i>30 \mathrm{~km} \mathrm{~s}^{-1}$.

\subsection{Comparison with published radial velocities}

Our radial velocities were compared to those already published. We have used the homogeneous radial velocities published by Andersen \& Nordström (1983) and Nordström \& Andersen (1985), quoted AN in Table 3, and those published in the WEB (Duflot et al. 1995) and Barbier-Brossat \& Figon (1998) catalogues quoted $\mathrm{B}$ in Table 3. In all cases, variable radial velocities are excluded as well as those whose discrepancy $(d v)$ with our results exceeds $8 \mathrm{~km} \mathrm{~s}^{-1}$. Moreover, in the WEB and Barbier-Brossat \& Figon catalogues, we have eliminated those with less than 3 measurements and an error greater than $2.2 \mathrm{~km} \mathrm{~s}^{-1}$. 138 common stars remained with AN and 164 with B. Our results are more reliable, probably due to a better resolution, but unfortunately have often only one measurement making an eventual undetectable binary (stars having a published radial velocity have been observed only to obtain $v \sin i$ ). As can be seen in Table 3 , the average of $d v$ is close to zero showing that our radial
Table 3. Comparison with published radial velocities

\begin{tabular}{llll}
\hline Source & $\overline{d v}$ & rms & $n$ \\
\hline AN & 0.09 & 0.65 & 138 \\
B + WEB & 0.04 & 0.54 & 164 \\
\hline
\end{tabular}

velocities are statistically in the same system as the literature. The independence of $\overline{d v}$ from the spectral type has been verified with AN.

\section{Results}

\subsection{Description of tabular results}

Individual velocity measurements are given in Table 7 (available in electronic form only; see footnote to title page). The columns are: HIP and HD numbers, heliocentric Julian Day for the middle of the integration time JJ, radial velocity $v_{i}$ and its error $\sigma_{i 0}, T_{\text {eff }}$ and rotation parameters of used synthetic spectrum and difference $d v_{\mathrm{gp}}$ between Gaussian and parabolic fitting.

When there are several observations for the same star, the average of the radial velocities $\bar{v}$ and its $\mathrm{rms} \sigma_{\mathrm{e}}$ are computed.

$\sigma_{\mathrm{e}}=\sqrt{1 /(n-1) \sum\left(v_{i}-\bar{v}\right)^{2}}$.

Table 8 (available in electronic form only; see footnote to title page) gives in order, the HIP and HD numbers, ICRS coordinates (consistent with J2000) from the Hipparcos catalogue, $V$ (Johnson) magnitude and its source from the Hipparcos catalogue ("G": ground-based photometry, "H": from $H_{\mathrm{p}}$ and a colour index, "T": from Tycho photometry), the spectral type from the CDS (Centre de Données Astronomiques de Strasbourg), the mean radial 
Table 4. Meaning of the flag (Cols. 106-107 of Table 8 and 58-59 of Table 9)

\begin{aligned} & \hline code Meaning \\ & \hline 0 symmetric and Gaussian peak \\ & 1 SB2 \\ & 2 certain double, near spectral types \\ & 3 certain double, A-B type with faint F-G component \\ & 4 probable double \\ & 5 suspected double \\ & 6 probable multiple system \\ & 7 certain shell star \\ & 8 suspected shell star \\ & 9 wide and irregular peak \\ & 10 wide peak of B star (few lines) \\ & \hline\end{aligned}

Table 5. Stars with no radial velocity. "R" refers to Table 6

\begin{tabular}{rrrl}
\hline HIP & HD & ST & note \\
\hline 11407 & 15371 & B5IV & \\
16339 & 21981 & A1V & \\
28855 & 41759 & A1V & \\
37752 & 62893 & B7V & \\
72192 & 129791 & A0V & \\
95793 & 183324 & A0V & R \\
114132 & 218242 & A1V & \\
117452 & 223352 & A0V & R \\
\hline
\end{tabular}

velocity $\bar{v}$, its rms $\sigma_{\mathrm{e}}$, its error $\epsilon_{\mathrm{e}}=\sigma_{\mathrm{e}} \sqrt{n}$, the number of observations $\mathrm{n}$, the mean adopted internal error $\sigma_{i}$, the ratio $E / I$ of external to internal error, the indication of radial velocity variability (Chi-square test), a number function of the shape of the peak obtained by correlation with minimum rotation and described in Table 4, "R" if a remark function of the object is given in Table 6 and finally Hipparcos flags of possible duplicity as described in Sect. 4.2.3.

\subsection{Binary and multiple stars}

An important number of suspected binaries are found in our sample by the shape of the peak. A lot of these binaries are physical, but there are optical systems also. So it is important to compare with two other criteria: the variability test upon the radial velocity and the data of Hipparcos mission. It is obvious that the conditions of detection are not the same for the three criteria. In fact they are complementary in terms of separation, difference of magnitude and orbital period. When the shape of the correlation peak is the only investigation mean, systematic simulations help in explaining the system of components.
Table 6. Notes about peculiar and multiple stars, these last stars belonging to the MSC (catalogue of physical multiple stars of Tokovinin 1997). $\lambda$ Boo-type stars are taken in Paunzen et al. 1997 (1), Gray 1997 (2) and Abt \& Morell 1995 (3)

\begin{tabular}{lll}
\hline HIP & HD & note \\
\hline 636 & 319 & $\lambda$ Boo-type star $(1,2)$ \\
8593 & 11413 & $\lambda$ Boo-type star $(1,2,3)$ \\
11261 & 15004 & shell with asymmetry of the peak \\
18275 & 24863 & shell with asymmetry of the peak \\
22192 & 30422 & $\lambda$ Boo-type star $(1,2)$ \\
22845 & 31295 & $\lambda$ Boo-type star $(1,2,3)$ \\
26865 & 38090 & shell with asymmetry of the peak \\
27341 & 38735 & 3 components \\
27713 & 39421 & $\lambda$ Boo-type star $(1)$ \\
33705 & 52516 & shell with asymmetry of the peak \\
34617 & 54958 & 3 components \\
37843 & 62864 & 3 components \\
39794 & 68520 & 3 components \\
41361 & 71487 & 4 components \\
41893 & 72462 & 3 components \\
42913 & 74956 & 4 components \\
43354 & 75654 & $\lambda$ Boo-type star $(1)$ \\
45167 & 79108 & $\lambda$ Boo-type star $(3)$ \\
49321 & 87344 & 3 components \\
50520 & 89715 AB & two stars on the slit \\
52520 & 93344 & 3 components \\
61463 & 109557 & 3 components \\
62788 & 111786 & $\lambda$ Boo-type star $(1,2,3)$ \\
63414 & 112846 & 3 components \\
76143 & 138488 & 3 components \\
77660 & 141851 & $\lambda$ Boo-type star $(1)$ \\
78078 & 142703 & $\lambda$ Boo-type star $(1,2,3)$ \\
79081 & 144667 & 5 components and accretion disk \\
87813 & 163336 & 3 components \\
90806 & 170680 & $\lambda$ Boo-type star $(1,2,3)$ \\
95793 & 183324 & $\lambda$ Boo-type star $(1,2,3)$ \\
98038 & 188405 & 3 components \\
100288 & 193281 & 4 components, $\lambda$ Boo-type star $(1,2)$ \\
105819 & 204041 & $\lambda$ Boo-type star $(1,2,3)$ \\
109306 & 210111 & $\lambda$ Boo-type star $(1,2)$ \\
117452 & 223352 & $\lambda$ Boo-type star $(3)$ \\
\hline & &
\end{tabular}

The width of the slit is $1.5^{\prime \prime}$ on the sky. So when the separation $\rho$ of components is higher than $2^{\prime \prime}$, the star is always seen as single. Using the Chi-Square test, the probability of variability with a confidence level of $99 \%$ was computed. In these cases, this information is given in Cols. 102-104 by "var" in Table 8. This test of variability depends on the dispersion of radial velocities around the mean value and the internal error, i.e. $T_{\text {eff }}, v \sin i$ and possible peculiarity of the star. 


\subsubsection{Non Gaussian peaks}

The interpretation of the shape of the peak is carried out by simulations as seen in Sect. 2.5. Detection of a binary depends on difference of radial velocities between the two components, difference of parameters $T_{\text {eff }}$ and $v \sin i$ and ratio of their intrinsic luminosities. Figure 1 gives an example of the simulation of a binary peak: $T_{\text {eff }}$ are 8500 and $6500 \mathrm{~K}$ and rotation 100 and $10 \mathrm{~km} \mathrm{~s}^{-1}$ respectively, their ratio of intensities is 22.2 and their difference of radial velocities is $32.07 \mathrm{~km} \mathrm{~s}^{-1}$. A faint late-type component is detectable in an early-type peak until a difference of magnitude of 4.5. So with propitious conditions, a component G0V can be detected on the peak of a A0-type star with rotation $200 \mathrm{~km} \mathrm{~s}^{-1}$. First a little tip is seen on the top of the peak, then it is replaced by an asymmetry at the bottom if the ratio of their luminosities increases. If the two components have close parameters, detection depends on their rotation and radial velocities difference essentially. First a deformation of the top of peak is obvious, then it is replaced by a bulging of sides if their rotation increases.

If the faint secondary peak was well defined, its radial velocity was measured by correlation with a cool synthetic spectrum ( $T_{\text {eff }}=6000 \mathrm{~K}$ and low rotation) and is quoted "b" in Table 7 after the HD number. It has been verified that the lunar light was not responsable. When peaks are well separated, the two are measured and results are given in Table 7, the most important peak being quoted the component "a". The average radial velocity is not computed and the HIP and HD numbers, coordinates, $V$ magnitude, the flag for the peak shape and the flags of Hipparcos catalogue are given in Table 9 , as in Table 8.

\subsubsection{Detected binaries by Hipparcos}

Hipparcos Catalogue (ESA, 1997) gives useful indications about binaries. The satellite measured more 24000 solved or suspected double (or multiple) systems 3000 of which being new with a separation $\rho<1^{\prime \prime}$ generally. Lindegren (1997) gives the conditions of detection which are very different from the spectroscopic ones. A binary system could be resolved if $\rho$ is between 0.1 and $30^{\prime \prime}$, $\Delta H_{\mathrm{p}}<4 \mathrm{mag}, H_{\mathrm{p}}$ being the Hipparcos magnitude and the period $P<30$ years with some difficulties if $\rho<0$ ". 4 or $\Delta H_{\mathrm{p}}>3.5 \mathrm{mag}$. The detection could result from photometric or astrometric data, generally both. If the photocenter deviated from the uniform motion expected for a single star, it is a suspected binary with an invisible companion. These indications are given in Tables 8 (and 9) respectively in the columns (bytes):

111(and 63): D indicates a probable binary detected by the Hipparcos photometry.

114 (and 66): the number of astrometric components.

116 (and 68): a double and multiple system flag about astrometry.
- C: resolved system $(P>3.3$ years $)$.

- O: orbital solution.

- G: solution with acceleration term (unresolved system, probable $P>10$ years).

- $V$ : variability of one of the components.

- X: stochastic solution (probable binary of $P<3$ years).

118 (and 70): solution quality flag (A: good, B: fair, C: poor, D: uncertain, S: suspected non-single).

120-1 (and 72-3): the concerned components.

123-129 (and 75-81): separation $\rho^{\prime \prime}$.

131-135 (and 83-87): difference of magnitude $\Delta H_{\mathrm{p}}$.

136 (and 88): an asterix indicates that $\rho^{\prime \prime}$ and magnitude difference are from CCDM (Dommanget \& Nys 1994).

More details are given in Hipparcos Catalogue Vol.1, Chap. 1.4.

These three criteria can be complementary. Taking into account only stars with variable radial velocities and detected as double by Hipparcos, the minimum binaries percentage is $60 \%$. If the criterion given by the shape of peak is added, this percentage increases to $74 \%$ with probable doubles or multiples (codes 4 and 6 of Table 4 ) and reaches $88 \%$ with suspected doubles (code 5 of Table 4 ), noticing these last percentages include optical systems.

Two spectroscopic binaries (HIP 33077 and 110838) have been found by McAlister et al. (1993) and are not binaries in Hipparcos Catalogue.

\section{Conclusion}

Radial velocities have been determined for 581 B8 to F2 stars, 159 being new. A detailed analysis of the A0 type stars for a detailed study of the HR-diagram (Gerbaldi et al. 1998) confirms the important percentage of binaries. The determination of $v \sin i$ has been made by Fourier transform and will be published soon (Royer et al.). A systematic comparison between his values and the rotation broadening of the adopted synthetic spectrum for each star shows that some of the stars with large $v \sin i$ are multiple systems in fact, and it confirms the preliminary results obtained with the Elodie spectrometer at the Haute Provence Observatory (Grenier et al.).

Acknowledgements. We would like to thank ESO staff members and night assistance of La Silla for their skillfulness and for their kindness.

\section{References}

Abt H.A., Morell N.I., 1995, ApJS 99, 135

Andersen J., Nordström B., 1983, A\&AS 52, 471

Barbier-Brossat M., Figon P., 1998, A\&AS (in press)

Burnage R., Gerbaldi M., 1990, 2nd ESO/ST-ECF Data Analysis Workshop, Baade D., Grosbøl P. (eds.). ESO Garching, p. 137 
Burnage R., Gerbaldi M., 1992, 4th ESO/ST-ECF Data Analysis Workshop, Baade D., Grosbøl P. (eds.). ESO Garching, p. 159

Dommanget J., Nys O., 1994, Comm. Obs. Royal de Belgique, Série A, No. 115

Duflot M., Figon P., Meyssonnier N., 1995, A\&AS 114, 269

ESA, The Hipparcos Catalogue, 1997, ESA SP-1200

Gerbaldi M., Gómez A.E., Faraggiana R., et al., 1989, The Messenger 56, 12

Gerbaldi M., Faraggiana R., Burnage R., et al., 1998, A\&A (submitted)

Gilliotte A., Lindgren H., 1989, ESO Operating Manual 7

Gray R.O., 1997, Schenectady Colloquium, Blue Stars (in press)

Grenier S., Royer F., Faraggiana R., et al., 1998 (in preparation to $\mathrm{A} \& \mathrm{~A})$
Kurucz R.L., 1993, CD-Rom 18

Latham D.W., Stefanik R.P., 1991, Trans. IAU 21B, 269

Lindegren L., 1997, Hipparcos Venice'97, ESA SP-402, p. 13

McAlister H.A., Mason B.D., Hartkopf W.I., et al., 1993, AJ 106,1639

Nordström B., Andersen J., 1985, A\&AS 61, 53

Paunzen E., Weiss W.W., Heiter U., et al., 1997, A\&AS 123, 93

Royer F., Gómez A.E., Gerbaldi M., et al., 1998 (in preparation to A\&A)

Sabas V., 1997, Thesis, Observatoire de Paris, France

Sabas V., Gómez A.E., Lebreton Y., et al., 1998 (submitted to A\&A)

Tokovinin A.A., 1997, A\&AS 124, 75

Tonry J., Davis M., 1979, AJ 84, 1511 